\title{
A case of nivolumab-induced cervical lymphadenopathy in a patient with gastric cancer
}

\author{
Koichi Taira, Akie Kimura, Akinobu Nakata, Yuji Nadatani, Shusei Fukunaga, Koji Otani, \\ Shuhei Hosomi, Fumio Tanaka, Noriko Kamata, Yasuaki Nagami, Toshio Watanabe, Yasuhiro Fujiwara \\ Department of Gastroenterology, Osaka City University Graduate School of Medicine, Osaka, Japan \\ Correspondence to: Koichi Taira. Department of Gastroenterology, Osaka City University Graduate School of Medicine, 1-4-3 Asahimachi, Abeno-ku, \\ Osaka City, 545-8585 Osaka, Japan. Email: koichit@med.osaka-cu.ac.jp.
}

\begin{abstract}
Nivolumab is a monoclonal antibody targeting programmed cell death-1 (PD-1) that has been recently shown to exhibit clinical efficacy in patients with gastric cancer. However, various degrees of immune-related adverse events (irAEs) have been reported. We report the case of a 71-year-old male patient diagnosed with gastric cancer with peritoneal metastases. He was treated with nivolumab as thirdline chemotherapy. On the $10^{\text {th }}$ day after completing seven cycles of nivolumab treatment, he urgently visited the hospital because of mild left cervical lymphadenopathy. We suspected it to be due to inflammation and initiated treatment with levofloxacin hydrate. However, 3 days later, he was admitted to the emergency room due to exacerbation of his lymphadenopathy. A diagnosis of nivolumab-induced lymphadenopathy was made as the antibiotics were ineffective, and the patient was administered prednisolone (PSL) $20 \mathrm{mg}$. One day after admission, the pain and swelling of the lymph node greatly lessened, and the following day, the pain gradually disappeared; thereafter, the PSL dose was tapered and nivolumab treatment was resumed. The patient again developed cervical lymphadenopathy approximately 4-5 days after nivolumab was reintroduced, which disappeared 1 week later. During each episode of lymphadenopathy, he received a dose of $20 \mathrm{mg}$ of PSL for 4 days, which would be eventually tapered to $10 \mathrm{mg}$ without antibiotics and NSAIDs. After 2 months, cervical lymphadenopathy completely disappeared while $10 \mathrm{mg}$ of PSL was continued, which was also eventually tapered off. To our knowledge, this is the first case report of nivolumab-induced lymphadenopathy in a patient with gastric cancer. This case suggested that we should keep in mind that various irAEs may occur during treatment with immune checkpoint inhibitors. It is necessary to ensure the absence of infection and metastasis before treatment and to promptly administer systemic corticosteroids to address them.
\end{abstract}

Keywords: Nivolumab; immune-related adverse events (irAEs); cervical lymphadenopathy; gastric cancer

Submitted Aug 13, 2020. Accepted for publication Nov 27, 2020.

doi: 10.21037/jgo-20-315

View this article at: http://dx.doi.org/10.21037/jgo-20-315

\section{Introduction}

Immune checkpoint inhibitors such as cytotoxic T-lymphocyte antigen 4, programmed cell death ligand 1 (CTLA-4), and programmed cell death-1 (PD-1) improve survival compared with standard chemotherapy and are widely used in multiple solid tumors. Nivolumab is a monoclonal antibody targeting PD-1, which has recently shown clinical efficacy in patients with gastric cancer (1).
In Japan, nivolumab was approved for gastric cancer as third-line or later treatment and has been widely used. Nivolumab has been reported to have few severe adverse events. However, specific immune-related adverse events (irAEs) occurred due to imbalances in immunological tolerance (2). Thus, it is important to recognize and manage rare specific irAEs.

In this report, we describe a case of cervical lymphadenopathy caused by nivolumab. To our 
knowledge, this is the first case report detailing the clinical course associated with such immune-related adverse lymphadenopathy. We present this case in accordance with the CARE reporting checklist (available at http://dx.doi. org/10.21037/jgo-20-315).

\section{Case presentation}

A 71-year-old male patient was referred to our hospital for further evaluation and management of gastric cancer with peritoneal dissemination. He had no notable medical history except for atrial flutter treated with pilsicainide hydrochloride hydrate. He received S-1 plus oxaliplatin as first-line chemotherapy and paclitaxel plus ramucirumab as second-line chemotherapy and achieved a partial tumor response, respectively. He was started on nivolumab as third-line chemotherapy because of the exacerbation of peritoneal dissemination.

On computed tomography (CT) imaging, his disease was found to be stable, and no nivolumab-induced irAE other than grade 2 thyroiditis was noted during the six cycles of nivolumab treatment he had received. On day 10 after completing seven cycles of nivolumab, he paid an urgent visit to the hospital because of mild, left lymphadenopathy and erythema from the lower jaw to the clavicle. We suspected an inflammatory etiology and started treatment with levofloxacin hydrate. However, 3 days after the consult, he was admitted to the emergency department because of trismus due to increasing lymph node size associated with pain and high fever. His body temperature, pulse rate, C-reactive protein (CRP) level, and white blood cell count were $38.4{ }^{\circ} \mathrm{C}, 78 \mathrm{bpm}, 7.86 \mathrm{mg} / \mathrm{dL}$ (normal range: $<0.40 \mathrm{mg} / \mathrm{dL}$ ), and $13,400 / \mu \mathrm{L}$ (normal range: 4,300 $8,000 \mu \mathrm{L}$ ), respectively. CT showed swelling of the left supraclavicular lymph node from the lower jaw (Figure 1). He was diagnosed with nivolumab-induced lymphadenopathy because the antibiotics proved ineffective. He received $20 \mathrm{mg}$ of prednisolone (PSL), diclofenac sodium, and piperacillin-tazobactam because infection could not be completely ruled out (Figure 2).

One day after admission, the pain and swelling of the lymph node improved. The pain gradually disappeared the next day; therefore, the PSL dose was reduced to $15 \mathrm{mg}$. Two days later, the CRP level decreased to $2.31 \mathrm{mg} / \mathrm{dL}$, and the PSL dose was further reduced and maintained at $10 \mathrm{mg}$. Nivolumab treatment was restarted after 6 days of discontinuation. However, cervical lymphadenopathy was noted to recur approximately 4-5 days after nivolumab was reintroduced, but this eventually disappeared 1 week later. During each episode of lymphadenopathy, he received a dose of $20 \mathrm{mg}$ PSL for 4 days, before it was reduced to $10 \mathrm{mg}$. After hospital discharge, the patient did not take any antibiotics or NSAIDs.

After 2 months, cervical lymphadenopathy completely disappeared while $10 \mathrm{mg}$ of PSL was continued, which was gradually tapered off (Figure 1).

No recurrence of cervical lymphadenopathy was observed even with the tapering of PSL dosage. Four months after discontinuing PSL, the patient stopped nivolumab treatment because of exacerbation of peritoneal dissemination; he was started on trifluridine/tipiracil as fourth-line chemotherapy. He had a partial tumor response during a total of 28 cycles of nivolumab treatment and did not experience further irAEs.

The authors are accountable for all aspects of the work in ensuring that questions related to the accuracy or integrity of any part of the work are appropriately investigated and resolved. All procedures performed in studies involving human participants were in accordance with the ethical standards of the institutional and/or national research committee(s) and with the Helsinki Declaration (as revised in 2013). Written informed consent was obtained from the patient.

\section{Discussion}

The mechanisms behind irAEs are still being elucidated. However, because of excessive immunity against normal organs, irAEs carry risks for pneumonitis, endocrine disorders, colitis, hepatitis, and neurologic adverse events (2). According to the ATTRACTION-2 study, a randomized phase 3 trial conducted to investigate the efficacy and safety of nivolumab for heavily treated advanced gastric or gastroesophageal junction cancer, allcause adverse events of any grade were reported in $91 \%$ of patients receiving nivolumab. However, as there were a few serious adverse events reported, treatment was discontinued in $3 \%$ of patients taking nivolumab (1). Generally, irAEs are effectively treated by systemic corticosteroids and additional immunosuppressants in more severe cases (3-5).

In our case, we diagnosed the patient with nivolumabinduced cervical lymphadenopathy because systemic corticosteroids were immediately effective where antibiotics were not. Furthermore, several studies have shown that recurrence of the same type of irAEs resulted in restarting of immune checkpoint inhibitor therapy in $20-43 \%$ of 

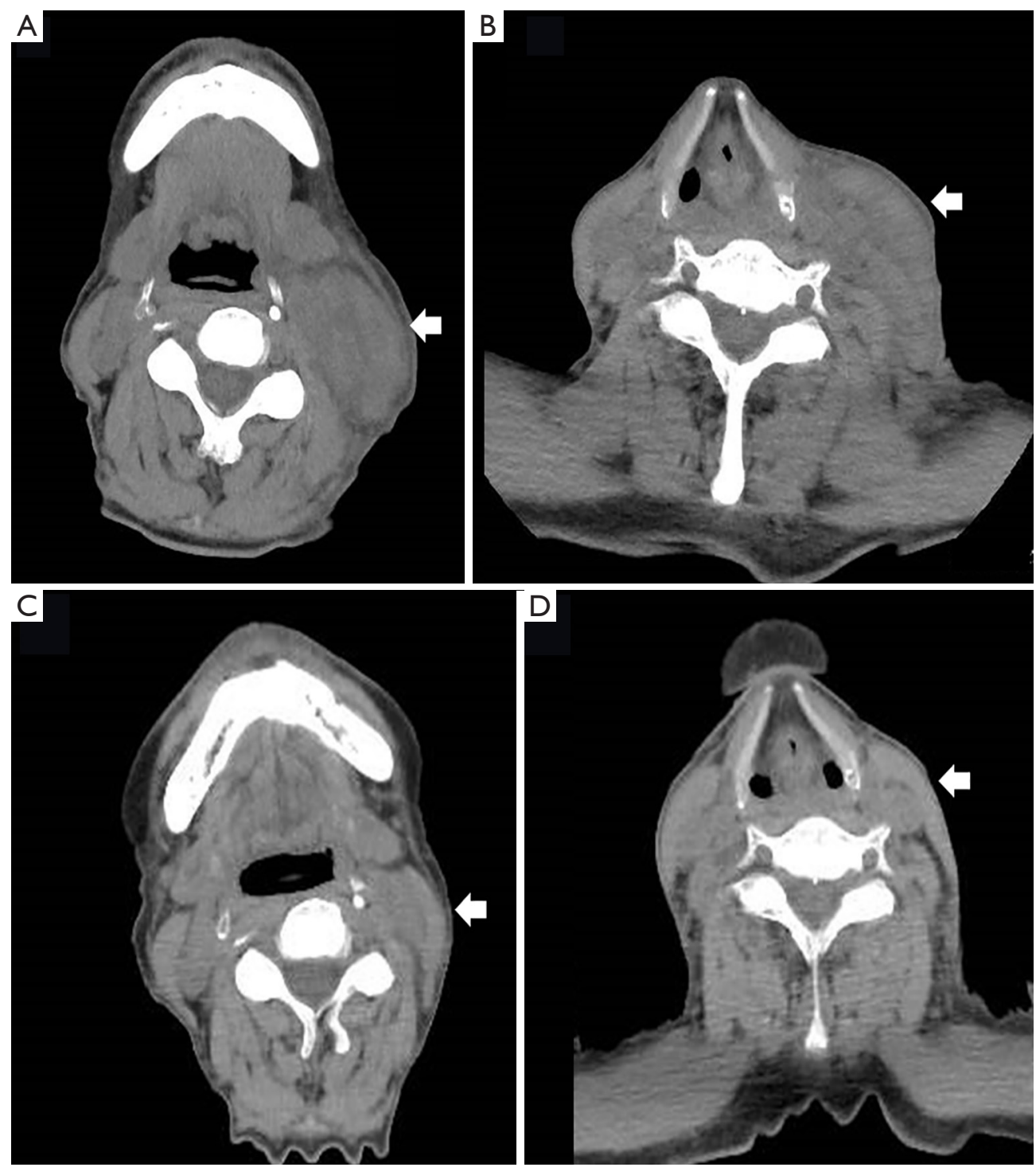

Figure 1 Computed tomography shows the appearance of cervical lymphadenopathy at the time of emergency hospitalization (A and B) and its complete disappearance after 2 months (C and D).

patients $(6,7)$. In our case, every time the patient took nivolumab for 2 months, cervical lymphadenopathy reoccurred, but this gradually disappeared when systemic corticosteroids were administered. Bacterial or viral etiologies of lymphadenopathy do not explain this recurrence. Moreover, the patient did not take antibiotics after the first episode. It may have been better to perform biopsy to confirm nivolumab-induced cervical lymphadenopathy. Furthermore, because of the recurrent lymphadenopathy and normal thyroid function, we could rule out the emergence of new sites of metastasis and thyroiditis. It was important to consider this as nivolumabinduced lymphadenopathy because if it was diagnosed as a new site of metastasis, then the treatment strategy would have been different. Benign sarcoid-like lymphadenopathy induced by an immune response to anti-CTLA- 4 therapy in melanoma patients has been reported by Berthod et al. (8). However, unlike our report, their lymphadenopathy was bilateral, symmetric hilar and mediastinal and resembled typical sarcoidosis.

In conclusion, we report the case a patient with gastric cancer who was treated with an immune checkpoint inhibitor 


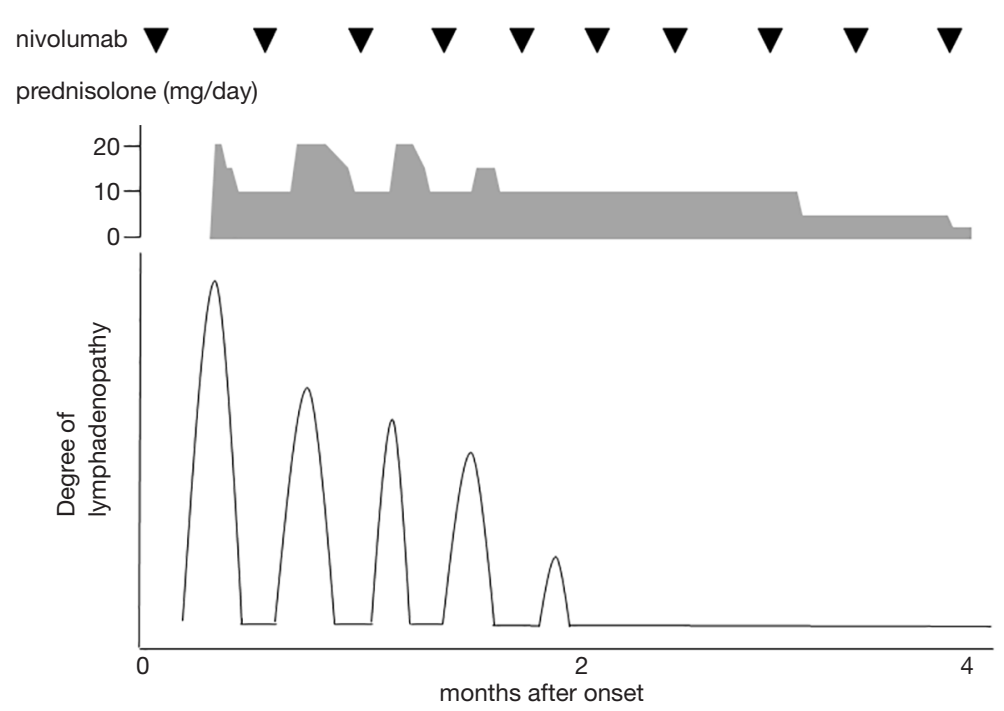

Figure 2 Clinical course of the present case. The time of nivolumab and prednisolone treatment is indicated relative to days after onset of lymphadenopathy.

and developed nivolumab-induced cervical lymphadenopathy, which improved with systemic corticosteroid administration. In cases when lymphadenopathy develops in patients under treatment with immune checkpoint inhibitors, irAEs should be considered as a differential diagnosis. Our case also demonstrated that there are various irAEs and it is important to diagnose and manage them promptly.

\section{Acknowledgments}

The authors would like to thank the patient and his family for allowing us to share the details of his case.

Funding: None.

\section{Footnote}

Reporting Checklist: The authors have completed the CARE reporting checklist. Available at http://dx.doi.org/10.21037/ jgo-20-315

Conflicts of Interest: All authors have completed the ICMJE uniform disclosure form (available at http://dx.doi. org/10.21037/jgo-20-315). The authors have no conflicts of interest to declare.

Ethical Statement: The authors are accountable for all aspects of the work in ensuring that questions related to the accuracy or integrity of any part of the work are appropriately investigated and resolved. All procedures performed in studies involving human participants were in accordance with the ethical standards of the institutional and/or national research committee(s) and with the Helsinki Declaration (as revised in 2013). Written informed consent was obtained from the patient.

Open Access Statement: This is an Open Access article distributed in accordance with the Creative Commons Attribution-NonCommercial-NoDerivs 4.0 International License (CC BY-NC-ND 4.0), which permits the noncommercial replication and distribution of the article with the strict proviso that no changes or edits are made and the original work is properly cited (including links to both the formal publication through the relevant DOI and the license). See: https://creativecommons.org/licenses/by-nc-nd/4.0/.

\section{References}

1. Kang YK, Boku N, Satoh T, et al. Nivolumab in patients with advanced gastric or gastro-oesophageal junction cancer refractory to, or intolerant of, at least two previous chemotherapy regimens (ONO-4538-12, ATTRACTION-2): a randomised, double-blind, placebocontrolled, phase 3 trial. Lancet 2017;390:2461-71.

2. Postow MA, Sidlow R, Hellmann MD. Immune-Related 
Adverse Events Associated with Immune Checkpoint Blockade. N Engl J Med 2018;378:158-68.

3. Teraoka S, Fujimoto D, Morimoto T, et al. Early ImmuneRelated Adverse Events and Association with Outcome in Advanced Non-Small Cell Lung Cancer Patients Treated with Nivolumab: A Prospective Cohort Study. J Thorac Oncol 2017;12:1798-805.

4. Weber JS, D'Angelo SP, Minor D, et al. Nivolumab versus chemotherapy in patients with advanced melanoma who progressed after anti-CTLA-4 treatment (CheckMate 037): a randomised, controlled, open-label, phase 3 trial. Lancet Oncol 2015;16:375-84.

5. Weber JS, Hodi FS, Wolchok JD, et al. Safety Profile of Nivolumab Monotherapy: A Pooled Analysis of Patients

Cite this article as: Taira K, Kimura A, Nakata A, Nadatani Y, Fukunaga S, Otani K, Hosomi S, Tanaka F, Kamata N, Nagami Y, Watanabe T, Fujiwara Y. A case of nivolumab-induced cervical lymphadenopathy in a patient with gastric cancer. J Gastrointest Oncol 2021;12(2):880-884. doi: 10.21037/jgo-20-315
With Advanced Melanoma. J Clin Oncol 2017;35:785-92.

6. Simonaggio A, Michot JM, Voisin A, et al. Evaluation of Readministration of Immune Checkpoint Inhibitors After Immune-Related Adverse Events in Patients With Cancer. JAMA Oncol 2019;5:1310-7.

7. Abou Alaiwi S, Xie W, Nassar AH, et al. Safety and efficacy of restarting immune checkpoint inhibitors after clinically significant immune-related adverse events in metastatic renal cell carcinoma. J Immunother Cancer 2020 02;8:e000144.

8. Berthod G, Lazor R, Letovanec I, et al. Pulmonary sarcoid-like granulomatosis induced by ipilimumab. J Clin Oncol 2012;30:e156-9. 